\title{
Prostitución y trata con fines de explotación sexual en la prensa digital española: análisis comparativo de la producción informativa
}

\author{
Prostitution and trafficking in the Spanish digital press: \\ comparative analysis of news production
}

\section{Prostituição e tráfico para fins de exploração sexual na imprensa digital espanhola: análise comparativa da produção informativa}

Vanesa Saiz Echezarreta, Universidad de Castilla-La Mancha, Cuenca, España (vanesa.saiz@uclm.es)

Diana Fernández Romero, Universidad Rey Juan Carlos, Madrid, España (diana.fernandez.romero@urjc.es)

María Cruz Alvarado López, Universidad de Valladolid, Valladolid, España (mariacruz.alvarado@uva.es)

RESUMEN | Este trabajo propone el análisis comparativo de la información publicada sobre prostitución y trata con fines de explotación sexual en medios españoles en dos períodos, 2013-2014 y 2018, con muestras de 491 y 1122 piezas, respectivamente. La intención es identificar, mediante una triangulación metodológica que incluye el mapeo etnográfico, el análisis de contenido y el discursivo, los indicios y tendencias de la agenda mediática sobre prostitución y trata, los patrones de representación, los relatos dominantes y la importancia que adquieren acontecimientos que atañen a la regulación normativa, moral y política del fenómeno. Se concluye que se detectan continuidades en las temáticas, los relatos y los actores, manteniéndose la prioridad de las acciones policiales y judiciales. Cuantitativamente, la importancia de la información generada por asuntos polémicos es poco relevante, pero algunos de ellos marcan un cambio de tendencia y una ruptura con la continuidad informativa que permite la aparición de nuevos actores y relatos, como la perspectiva del trabajo sexual, que anteriormente recibía una cobertura anecdótica.

PALABRAS CLAVE: prostitución; trata con fines de explotación sexual; prensa digital; trabajo sexual; abolicionismo; representaciones sociales. 
ABSTRACT/In this paper we propose the comparative analysis of the information published on prostitution and trafficking for sexual exploitation in Spanish media in two periods, 2013-2014 and 2018, with samples of 491 and 1,122 pieces, respectively. The intention is to identify, through a methodological triangulation that includes ethnographic mapping, content analysis and discourse, the indications and trends that make up the media agenda on prostitution and trafficking, the patterns of representation, the dominant stories, and the importance that significant events they regarding legislative, moral and political regulation of this phenomenon. We conclude that there are continuities in the themes, stories and actors, maintaining the priority of the police and judicial actions. Quantitatively, the importance of the information generated by controversial issues is not very relevant, but some issues mark a change in the trend and a break with the information continuity that allows the appearance of new actors and stories, such as the perspective of sex work, which previously received anecdotal coverage.

KEYWORDS: prostitution; trafficking; digital media; sex work; abolitionism; social representations.

RESUMO | Este trabalho propõe a análise comparativa da informação publicada sobre prostituição e tráfico com fins de exploração sexual na mídia espanhola em dois períodos, 2013-2014 e 2018, com amostras de 491 e 1.122 peças respetivamente. O objetivo é identificar, através de uma triangulação de metodologia que contém o mapeamento etnográfico, a análise de conteúdo e discursivo, os indícios e tendências da agenda midiática sobre prostituição e tráfico, os padrões de representação, os relatos dominantes e a importância que adquirem os fatos que tem relação com a regulação normativa, moral e política do fenômeno. Conclui-se que são detetadas continuidades nas temáticas, os relatos e os atores, mantendo a prioridade das ações policiais e judiciais. De forma quantitativa, a importância da informação gerada por assuntos polêmicos é pouco relevante, mas alguns deles marcam uma mudança de tendência e uma ruptura com a continuidade informativa que permite aparecimento de novos atores e relatos, bem como a perspectiva do trabalho sexual, que antes recebia uma cobertura anedótica.

PALAVRAS-CHAVE: prostituição; tráfico com fins de exploração sexual; imprensa digital; trabalho sexual; abolicionismo; representações sociais. 


\section{EXPLOSIÓN DISCURSIVA DE LA CONTROVERSIA SOBRE PROSTITUCIÓN Y SU RELACIÓN CON LA TRATA}

Desde el punto de vista conceptual, la prostitución es abordada hoy desde múltiples perspectivas, en relación con diversos objetos de estudio: el sistema sexo/ género,el orden socio-sexual (Justo von Lurzer, 2014; Sabsay, 2016), el económico (Zelizer, 2011; Fraser, 2012) o los derechos humanos (Heim, 2012; Kempadoo, 2003; Kempadoo et al., 2005). Parece emerger un subcampo de investigación que ofrece "oportunidades para colaboraciones interdisciplinares que explican (no excusan) los modos en que los medios (...) están implicados en todas las facetas de la trata de personas", entre ellas, la explotación sexual (Friedman \& Johnston, 2018, p. 2).

Desde una perspectiva política, este asunto se articula cíclicamente como un problema de agenda sobre el que se reclaman medidas por parte de las instituciones, muy condicionado por la emergencia de una política transnacional sobre la trata y por el incremento de la preocupación por este delito. El desplazamiento hacia este asunto recupera y transforma las guerras del sexo, confrontando al neo abolicionismo y el movimiento pro trabajo sexual con la lucha contra la trata como telón de fondo (Kempadoo et al., 2005).

Los medios se han hecho eco de acciones generadas desde los distintos actores en liza: el movimiento feminista, las instituciones académicas, sociales y políticas, las agencias gubernamentales o los colectivos de mujeres (Bernstein, 2007).

\section{Controversia mediática y políticas públicas en la España reciente}

Desde la aprobación del Protocolo de Palermo (2000), se incrementó el interés mediático internacional por la trata con fines de explotación sexual. En España, desde 2008, se ha convertido en el enfoque principal de la cobertura sobre la prostitución. El primer estudio sistemático fue el de Calvo (2001); en 2010, Álvarez y sus colegas analizaron periódicos impresos gallegos; el primer estudio longitudinal aborda $\mathrm{El}$ País y ABC entre 1977 y 2012 (Puñal, 2015). Desde entonces, han aparecido estudios de caso con muestras menores (Gutiérrez García, 2013; Brosa \& Medina, 2012).

Las investigaciones anteriores a 2015 coinciden en la escasa presencia del tema en la agenda y en el enfoque tradicional caracterizado por la estereotipia, el lenguaje objetualizador, sensacionalista, morboso e irrespetuoso para con las mujeres, la representación ambivalente de estas como víctimas engañadas o delincuentes, un enfoque moral y estigmatizante, anclado en la lógica de sucesos, y la ausencia de actores (clientes y proxenetas) y de enfoques estructurales.

El paso al online y la aparición de la trata como problema público incrementan la producción de noticias y el interés académico. En torno a 2015 se produce un punto de inflexión a raíz de los siguientes estudios: Ayuntamiento de Madrid (2014), 
Ayuntamiento de Barcelona y Asociación de Mujeres periodistas de Cataluña (2015), Clua (2015), Puñal Rama y Tamarit (2017), Benítez-Eyzaguirre, (2017) o Saiz Echezarreta y Martínez Pérez (2018). Se apuntan algunos cambios en las representaciones debido a la priorización de la trata como asunto de agenda institucional y mediática, que desplaza en parte el discurso hegemónico (Núñez Puente \& Fernández Romero, 2015; Saiz Echezarreta et al., 2018). Del marco de la desviación y la amenaza, se ha evolucionado a otro más centrado en la criminalización.

La incorporación a la agenda mediática de la controversia sobre prostitución casi siempre está vinculada a acontecimientos de carácter legal, político y social. La Ley de Seguridad Ciudadana ${ }^{1}$ y la aprobación de ordenanzas municipales que recogían medidas para evitar la prostitución callejera ocasionaron una polémica recogida por los medios. En España la prostitución no está regulada, pero estas normativas abrieron la posibilidad de sancionar a los demandantes y fueron cuestionadas por no evitar las multas a potenciales víctimas de trata obligadas a ejercer en la vía pública.

La cuestión aparece en agenda cíclicamente, vinculada también con propuestas de actores políticos. De forma inédita, el partido Ciudadanos defendió la legalización en su programa para las elecciones generales (Piña, 2015). Las propuestas legislativas y la acción del gobierno son oportunidades para la cobertura mediática y la discusión pública del asunto, como el proyecto de ley para abolir la prostitución y el intento fallido de aprobar una ley de lucha integral contra la trata del gobierno socialista en 2018.

Esto surgió en respuesta a una acción del movimiento en defensa del trabajo sexual, que en agosto de 2018 logró que el Ministerio de Trabajo ${ }^{2}$ aprobase el sindicato Organización de Trabajadoras Sexuales (OTRAS), autorizado en el Boletín Oficial del Estado. Días después, el gobierno intervino para prohibirlo. La situación generó una fuerte polémica, en la que se posicionaron a través de los medios múltiples actores. En junio de 2021 los tribunales anularon esta decisión, reconociendo el derecho a crear el sindicato, aunque advirtieron que ello no implicaba legalizar la actividad en España.

Estudios académicos recientes (Saiz Echezarreta \& Martínez Pérez, 2018; Alvarado \& Von Luzer, 2019) muestran cómo estas acciones permiten incorporar en la agenda mediática a personas que se identifican como trabajadoras sexuales y a colectivos del movimiento, como el Sindicato OTRAS.

1. Ley Orgánica 4/2015 del 30 de marzo.

2. https://www.sindicatootras.org/ 
Desde el punto de vista político, se ha hecho más hincapié en diseñar e implementar planes de lucha contra la trata con fines de explotación sexual, a nivel nacional y autonómico. El II Plan Integral contra la trata con fines de explotación sexual (2015-2018) incluye como objetivo "la eliminación de imágenes estereotipadas y contenidos sexistas (...) que justifiquen, banalicen o inciten a la violencia contra la mujer, en particular, en relación con la incidencia de la demanda de servicios sexuales en la trata de mujeres y niñas con fines de explotación sexual" (Delegación del Gobierno Contra la Violencia de Género, 2015, p. 85). Esta medida ofrece un marco justificativo para el análisis.

\section{Estudios recientes de ámbito internacional}

Los estudios internacionales más recientes analizan cómo las representaciones mediáticas desempeñan un rol en el diseño, aprobación e implantación de normativas, nacionales o locales, y las actitudes y valores en relación con el tráfico y la trata. El objetivo es comprender en el marco de la mediatización (Hepp, 2020) la interdependencia de las instituciones mediáticas con el resto de las entidades que intervienen en los mercados del sexo y en el fenómeno de la trata (Saiz-Echezarreta, 2019).

Los análisis comparados son numerosos: Krsmanovic (2018) relaciona medios ingleses, holandeses y serbios, mientras que Snow (2017) contrasta las coberturas de The Guardian, The New Times y CNN. Otros se centran en la representación del trabajo sexual, donde está legalizado o descriminalizado, como en Grecia (Sagredos, 2017) o Nueva Zelanda (Easterbrook-Smith, 2018), si bien la mayoría aborda la representación de la trata o de la prostitución como marco más general. Algunas aproximaciones provienen de la Federación Rusa y estados balcánicos (Sobel, 2016; Bozorova, 2019; Rusnac, 2019), Italia (Di Ronco, 2017), Francia (Devillard \& Le Saulnier, 2015), Canadá (Strega et al., 2014), Sudáfrica (Hunt \& Hubbard, 2015) o Reino Unido (Papadouka et al., 2016; Gregoriou \& Ras, 2018). En Estados Unidos encontramos los trabajos de Austin y Farrell (2017) o el análisis longitudinal de Sanford y colegas, (2016) que compara una muestra de The New Times y Washington Post de 2012-2013, con otra de 1980-2006.

Estas investigaciones señalan el carácter reduccionista y sensacionalista de las coberturas y la focalización sobre las mujeres. Esto refuerza su estigmatización, con estrategias retóricas como la criminalización y la revictimización. Además, la exotización e hiper sexualización suele ser una estrategia de representación transversal, ligada al carácter marginal y transgresor de las sexualidades no heteronormativas. Las lógicas mediáticas circulan en representaciones clásicas estigmatizantes que vinculan principalmente prostitución con orden público, indecencia, y metáforas higienistas, al sobrerrepresentar la prostitución de calle. 
Los estudios recogen la aparición del movimiento en defensa del trabajo sexual y la consiguiente repolitización de la cuestión. También la persistencia del marco explicativo de la prostitución como problema asociado especialmente a la inmigración, y el encuadre del debate acerca de las soluciones en torno al eje abolicionismo/pro derechos. Esto se combina con cierta indeterminación y confusión en el uso de términos relacionados con la prostitución, el trabajo sexual y la trata (Papadouka et al., 2016). No obstante, se percibe una mejora en las coberturas periodísticas al precisar los conceptos y explicar los fenómenos, si bien las historias tienen excesiva dependencia de fuentes oficiales y priorizan exageradamente el enfoque criminológico, policial y judicial, y los asociados al rescate y la revictimización.

Aunque estos trabajos destacan que la prostitución y la lucha contra la trata han entrado en la agenda mediática y captan la atención pública (Austin \& Farrell, 2017), el asunto depende todavía en exceso de la agenda política (Krsmanovic, 2018). Así, puede ignorarse e invisibilizarse, si hay interés en hacerlo, o rebajar su presencia, para adecuarse a los procesos de diseño e implantación de políticas y normativas a diferentes niveles, como señala Allwood (2018) para la Unión Europea.

\section{HIPÓTESIS Y OBJETIVOS}

Alineándonos con el contex to internacional y conscientes de la influencia que la esfera mediática tiene en la sociedad, se considera necesario hacer un seguimiento del discurso periodístico sobre prostitución y plantear un estudio longitudinal contrastivo. Interesa saber si los medios mantienen una línea en el tiempo, en el contexto sociohistórico español.

Como hipótesis, se plantea que la introducción en los medios de la trata de mujeres y niñas con fines de explotación sexual, asumida como asunto público y político, está incidiendo en la representación de la prostitución en dos aspectos: polarizando el asunto y sesgándolo hacia posiciones ideológicas mayoritariamente abolicionistas, en las que trata y prostitución se identifican o asimilan, y construyendo un nuevo relato sobre el delito de trata, pero sin dejar de lado la tópica clásica sobre la prostitución.

El principal objetivo es analizar y contrastar el contenido publicado en la prensa digital española sobre prostitución y trata con fines de explotación sexual en dos períodos. Se estudiarán y compararán las noticias de una investigación realizada para el Ayuntamiento de Madrid del 15 de octubre de 2013 al 15 de marzo de 2014 para sondear la implicación de los medios durante la vigencia del II Plan municipal contra la explotación sexual y la atención a la prostitución (2013-2016). Por otro lado, se analizarán y contrastarán las noticias publicadas entre el 1 de junio y el 31 de diciembre de 2018, cuando finaliza el II Plan Integral estatal contra la trata (2015-2018) y la aprobación del sindicato OTRAS. 
Como objetivos específicos se establecen:

- Identificar qué asuntos componen la agenda mediática sobre prostitución y trata en España. Observar la importancia en términos cuantitativos y cualitativos de la información acerca de la controversia y el debate sobre prostitución generada mediante asuntos polémicos (aprobación del sindicato OTRAS), frente al resto de información cotidiana y de continuidad sobre el tema (campañas, acciones policiales y judiciales, manifestaciones políticas, discusión ciudadana, etc.).

- Explorar de modo cualitativo los patrones de representación, los ejes temáticos, las voces que se priman, los imaginarios y las líneas discursivas dominantes a nivel nacional, regional y local.

- Describir las variaciones significativas al respecto en ambos períodos.

\section{METOdOLOGÍA}

Este estudio se inscribe en una investigación longitudinal más amplia, con foco en la mediatización de la controversia sobre prostitución en el espacio público entre 2014 y 2020. Se basa en aproximaciones etnográficas a diferentes arenas públicas en las que se produce este debate, especialmente las mediáticas digitales, y se reconoce en la propuesta de mapeo de controversias públicas (Guerrero Bernal et al., 2018; Venturini, 2012; Marres, 2015, 2017).

A partir de diversos estudios de caso, confirmamos que las lógicas mediáticas afectan performativamente tanto a las dinámicas de la deliberación, como a la definición del marco legal, al diseño de las políticas públicas y a los saberes y creencias compartidas en torno al tema.

En este contexto, los medios son espacios de discusión y actores interesados, con voz propia en el debate. Analizar el discurso informativo reciente da acceso a los procesos en que los actores implicados se confirman y legitiman.

Recurrimos a la triangulación metodológica, "un proceso de contraste entre las técnicas de investigación que permite comparar y completar los resultados de cada una de ellas sobre un objeto de estudio común, con el objetivo de perfeccionar la validez y la fiabilidad del conjunto del trabajo" (Vicente Mariño, 2009, p. 5). Si el mapeo etnográfico de la controversia permite identificar actores, escenarios y perspectivas en el proceso de publicitación del asunto (Cefaï, 2016), combinar el análisis de contenido y el de discurso facilita identificar patrones representacionales en el discurso informativo.

Para obtener los datos se aplica un análisis de contenido cuantitativo de la producción periodística, interpretados con técnicas cualitativas derivadas del análisis de discurso con perspectiva semiótica, paraanalizar sus aspectos formales, fuentes y temáticas, implementación a nivel nacional y regional y su evolución. 
No obstante, la etapa cuantitativa no busca una representatividad estadística, sino reconstruir una indicialidad productiva (Souza \& Carvalho, 2012). Cuantificar es una guía para indagar en la complejidad mediante una visualización exploratoria, lo que se identifica como un análisis de contenido que incluye una definición inductiva del sistema de categorías a partir de las lecturas e interpretación del material (Kohlbacher, 2006; Souza \& Carvalho, 2012).

Se realizó un análisis comparativo de dos muestras. La primera incluye información publicada en la prensa digital española entre junio y diciembre de 2018: 1122 piezas de 108 medios de comunicación de 17 comunidades autónomas. Comprende los diarios de los principales grupos de comunicación españoles y los de mayor relevancia en cada comunidad autónoma según los índices de la Oficina de Justificación de la Difusión (OJD) ${ }^{3}$ y fue recopilada por un equipo de 28 investigadores/as en el marco de una subvención del Instituto de la Mujer"

3. Los medios analizados son: Andalucía: Diario Sur; Ideal de Almería; Ideal de Granada; Ideal de Jaén; Ideal de Sevilla; Ideal de Andalucía; La voz de Almería; Viva Jaén - Andalucía Información; Diario Córdoba, Diario Jaén; Huelva Ya; Diario de Sevilla; Málaga Hoy; Diario de Almería; Huelva Información; Granada Hoy; Diario de Cádiz (17). Aragón: El diario de Alto Aragón, Diario de Teruel, Aragón Digital, Heraldo (4). Asturias: El Comercio(Diario de Asturias) (1). Cantabria: El Faradio; Diario de Cantabria; El Diario Montañés (3). Castilla-La Mancha: El digital de Albacete; El digital Castilla-La Mancha; Voces de Cuenca; Lanza Digital; Guadalajara Diario; La Tribuna de Cuenca (6). Castilla y León: Diario de Ávila; Diario Palentino Digital; El Heraldo de Soria, El Día de Segovia, El Adelantado de Segovia, Diario de Burgos.es, Burgosconecta.es, Burgosnoticias, Zamora24horas, Zamoranews, La Opinión de Zamora, El Día de Valladolid, El Diario de Valladolid, Tribuna de Valladolid, Diario de León, León noticias, ileon.com, Digitalleón.com, Ahora León, salamanca24horas.com, lagacetadesalamanca. es, La Crónica de Salamanca, salamancartvealdia.es, Noticias cyl salamanca, Noticias cyl Valladolid, El Norte de Castilla (26). Cataluña: La Vanguardia, El Periódico; Naciodigital, Catalunyadiari; Ara. cat; Vilaweb; Elnacional.cat; El mon; Diari de Girona; El Punt Avui; Grup Raco Català; Regió 7; Segre. com; El 9 NOU; Diari Mes; Diari La Veu; Eixdiari; Emporda; El Critic; Ebre Digital; Lleida.com (21). Comunidad Valenciana: Levante, Las Provincias, Diario Información, La Veu (4). Extremadura: El Periódico Extremadura; La Crónica Badajoz; Hoy (3). Galicia: La Voz de Galicia, Faro de Vigo. (2). Islas Baleares: Ara Baleares, Diario de Mallorca, Última Hora (3). Islas Canarias: Canarias 7(1). La Rioja: Rioja2, Noticias de la Rioja, Nuevecuatrouno (3). Madrid / Generalistas: ABC, El diario.es, El País, El Confidencial, El Español, El Mundo (6). Navarra: Diario de Navarra, Diario de noticias (2). País Vasco-Euskadi: Berria; Deia; El Correo; Gara (4). Región de Murcia: La Opinión, La Verdad (2). 4. Proyecto Simposio: La representación de la trata con fines de explotación sexual en el espacio público. PAC18 exp.:29/2ACT/18. Convocatoria competitiva IMIO: subvenciones para realizar postgrados de estudios de género y actividades del ámbito universitario relacionadas con la igualdad de oportunidades entre mujeres y hombres (2018). Instituto de la Mujer y para la Igualdad de Oportunidades, Ministerio de la Presidencia, Relaciones con las Cortes e Igualdad. 
Categorías de identificación

Comunidad autónoma

Fecha

Medio

Relevancia

URL

Titular

Subtítulo

Género periodístico

Noticia breve, noticia, reportaje, crónica, entrevista, editorial, vídeos.

Autoría

Hombre, mujer, agencia, medios, otros.

$\mathrm{N}^{\circ}$ comentarios

Facebook

Twitter

Etiquetas

Sección

Sociedad, política, economía, sucesos, cultura, local/regional, deporte, otros.

\section{Categorías de carácter temático}

\section{İndices}

Acciones de ONG especializadas, judiciales, legales, policiales,

Temática

de sensibilización, activismo abolicionista, activismo

trabajo sexual, declaraciones institucionales, información

sociocultural, información estadística e informes, testimonios en primera persona, otros contextos y otros.

Sindicato OTRAS

Demanda como centro

Proxenetismo como centro

Superviviente

Distinción del fenómeno

Otras prostituciones

Fuentes
Alude o no al sindicato.

Pone en el centro la demanda.

Pone en el centro el proxenetismo.

Pone en el centro a las mujeres como supervivientes.

Se abordan la trata y la prostitución como fenómenos diferentes o similares.

Se contemplan otros tipos.

Colectivo migratorio mencionado

Categorías de análisis visual

Índices

Mujeres pixeladas, de espaldas, colectivos de mujeres,

Fotografías demandantes de prostitución, detenidos enjuiciados, agentes institucionales, fuerzas y cuerpos de seguridad, ilustraciones.

Vídeos

Vídeo noticia, vídeo entrevista, vídeo policial, vídeo campaña, otras.

Otros elementos

Tabla 1. Retícula para identificar las unidades de análisis y de su contenido verbal y visual

Fuente: Elaboración propia. 
La búsqueda se realizó en las hemerotecas de los medios digitales con la palabra clave prostitución, concepto idóneo por abarcar la información publicada tanto de prostitución como de trata con fines de explotación sexual, dado que estudios precedentes evidencian la conexión que realizan los medios entre ambas.

Por otro lado, se recurrió al trabajo promovido por el Ayuntamiento de Madrid (2014) que recopiló 491 noticias con la palabra clave prostitución entre el 15 de octubre de 2013 y el 15 de marzo de 2014 en nueve medios españoles 5 .

La segunda muestra (2018) adaptó una retícula de análisis de contenido cuantitativo preexistente, testada en dos investigaciones previas (Ayuntamiento de Madrid, 2014), que dio lugar a la muestra previa de contraste y la utilizada en un trabajo del Ayuntamiento de Barcelona y la Asociación de Mujeres Periodistas de Cataluña (2016).

Se utilizaron 25 categorías, 14 para identificar cada unidad de análisis y el resto de carácter temático, referidas a su contenido verbal y visual.

En cada comunidad autónoma un investigador/a coordinó la información recopilada. Posteriormente un equipo de cuatro personas procedió a una revisión general para homogeneizar los datos generales. Los registros pasaron una doble revisión, que eliminó las noticias repetidas y pulió los resultados de la codificación.

\section{RESULTADOS}

Se ofrecen los resultados más relevantes del análisis comparativo entre los patrones de representación de la prostitución y la trata por parte de los medios nacionales, regionales y locales en los años 2013-2014 y 2018, según contenidos informativos, relatos y actores, y pautas de representación visual.

\section{Contenidos informativos}

El estudio de la muestra de 2013-2014 (491 piezas) concluye que los nueve medios analizados incluyen con asiduidad noticias sobre casos de trata con fines de explotación sexual y relacionados con la prostitución. La recopilación de 1122 piezas entre junio y diciembre de 2018 en 108 medios de comunicación de todo el territorio nacional permite inferir una tendencia similar, con matices. Podemos concluir que, si bien el tema se ha consolidado en la agenda nacional, no ha ocurrido así en el ámbito regional y local, porque la información sigue siendo muy dependiente de las agencias.

5. Diarios ABC, El País, El Mundo, 20 Minutos, Público, Marca; cadenas RTVE, Telemadrid y revista Interviú. 
En el estudio de 2014 los porcentajes de noticias procedentes de agencia oscilan entre el 78,18\% de 20 Minutos y el 5\% de El País, con una media de 26,28\%. Las piezas firmadas por redacción suponen un 10,71\%.

En la muestra 2018 se contabilizaron un $23,98 \%$ de las piezas firmadas por agencia y un $16,04 \%$ por el medio. Se observa una tendencia similar con respecto a la homogeneidad derivada de la escasa especialización y de la utilización de los relatos que proporcionan las agencias.

Los datos en ambas muestras indican que existe casi un equilibrio entre las piezas firmadas por hombres y mujeres. En el estudio de 2014 se detecta que aquellas que presentan mayor especialización y profundidad son firmadas mayoritariamente por mujeres, que en ocasiones adoptan una perspectiva de género. En la muestra de 2018 hay un porcentaje ligeramente mayor de piezas, el $24,51 \%$, suscritas por periodistas hombres, frente al $21,93 \%$ firmadas por mujeres. Aunque cabría esperar un sesgo de género habitual en el ámbito de la opinión por la menor visibilidad de las firmas de mujeres, la participación es muy similar: del $17,74 \%$ de piezas de opinión publicadas, 4,46\% corresponde a hombres y 4,28\% a mujeres. En los reportajes hay mayor impronta de mujeres: 2,58\% frente a 1,87\%.

En ambas muestras se prioriza la información local y regional: 52\% de las piezas de 2013-2014 pertenece a la sección de información local, 22\% a la de nacional y $26 \%$ a la internacional. Prodigan las crónicas locales por encima de las noticias nacionales e internacionales, lo cual delata la escasa trascendencia atribuida a ambos asuntos en la agenda pública política, más allá de los hechos puntuales que acontecen relatados como sucesos localizados. Debido a las particulares características de la muestra de $2018,38,41 \%$ se inscribe en las secciones local/ regional. Las siguientes secciones donde se ubican más piezas son Sociedad $(20,14 \%)$, Otros (14,53\%), Sucesos (9,8\%) y Opinión (9,27\%). Estos datos aportan solo una aproximación al marco en el que se inscriben las piezas, teniendo en cuenta la pérdida de relevancia de las secciones en la prensa digital. Más interesante es que el grueso de ambas muestras corresponda a noticias breves y de extensión media. En las piezas de 2013-2014, 7\% es parte de la sección de opinión (33 artículos) y 5\% (26) puede categorizarse como reportajes o noticias de mayor longitud. En los meses seleccionados se suscitó un importante debate sobre la prostitución en España por la reforma del Código Penal, que modificaba la conceptualización y los supuestos sobre el proxenetismo, y la nueva Ley de Seguridad Ciudadana, que contemplaba un incremento en las sanciones por el ejercicio de prostitución callejera, incluyendo multas por la demanda. Ello ocasionó que los artículos de opinión prodigaran más que en otros momentos, lo cual nos permite comparar con el período de 2018 seleccionado a propósito del sindicato OTRAS. Lo que sorprendió entonces fue 
la escasísima presencia de reportajes o piezas más largas y de entrevistas. La información oficial, el discurso indirecto y la mención predominaron sobre los testimonios en primera persona, una tendencia que a partir de 2015 cambió con la presencia de la voz legitimada del movimiento en pro del trabajo sexual (Saiz Echezarreta \& Martínez Pérez, 2018).

En 2018 encontramos 61,41\% de noticias y 17,74\% de noticias breves publicadas. Entonces se difundió un 8,9\% de columnas de opinión (100 en total) y un 0,45\% de editoriales (cinco en total), además de un 4,9\% de reportajes (55), un 3,65\% de entrevistas (41) y un 1,78\% de crónicas (20). Llama la atención el elevado número de entrevistas y de reportajes con respecto al otro período.

La aprobación del sindicato OTRAS en agosto de 2018 y su ilegalización en noviembre incrementaron el debate en la esfera mediatizada. Los medios fueron altavoz de las dos posturas aparentemente irreconciliables: abolicionismo y movimiento pro trabajo sexual. Debido a que la navegación virtual resulta más flexible, se realizó la clasificación temática de las piezas para atender a su contenido. En la muestra de 2013-2014, 33\% corresponde a noticias sobre operaciones policiales y criminalidad, $19 \%$ versa sobre legislación y normativa, $9 \%$ son crónicas sobre clientes famosos y juicios y las historias de contextualización suponen un 13\%. Las temáticas de menor aparición son juicios (5\%), cultura y variedades (6\%) y opinión (7\%).

En la muestra de 2018 se recontó $22,46 \%$ de informaciones de acciones policiales, seguidas por $16,4 \%$ de acciones judiciales, $10,7 \%$ de acciones legales, $11,76 \%$ de acciones de sensibilización, 8,91\% de información sociocultural y 8,56\% de declaraciones institucionales. Destacan así sobre el resto las acciones policiales y judiciales y las acciones legales, en un contexto en el que está vigente la Ley de Seguridad Ciudadana. Sin embargo, se observan indicios de presencia de temas insertos en la agenda en un ámbito marcado por las acciones contra la trata y el debate sobre el sindicato OTRAS que facilitan un marco orientado a la política y a la acción de los movimientos sociales. Por eso, se encuentran piezas sobre acciones de sensibilización, información sociocultural, declaraciones institucionales, así como de activismo abolicionista y trabajo sexual (vinculado a la polémica por el sindicato).

En menor medida se publicó información estadística, testimonios en primera persona y acciones de ONG especializadas. Cabe señalar la importancia tanto de la publicación de los datos como de su ausencia y de su calidad, tanto en el abordaje del delito como en la propia controversia de la prostitución ${ }^{6}$.

6. Ver problemas derivados de la falta de datos y cómo lastran el debate a nivel internacional (Raphael, 2017). 


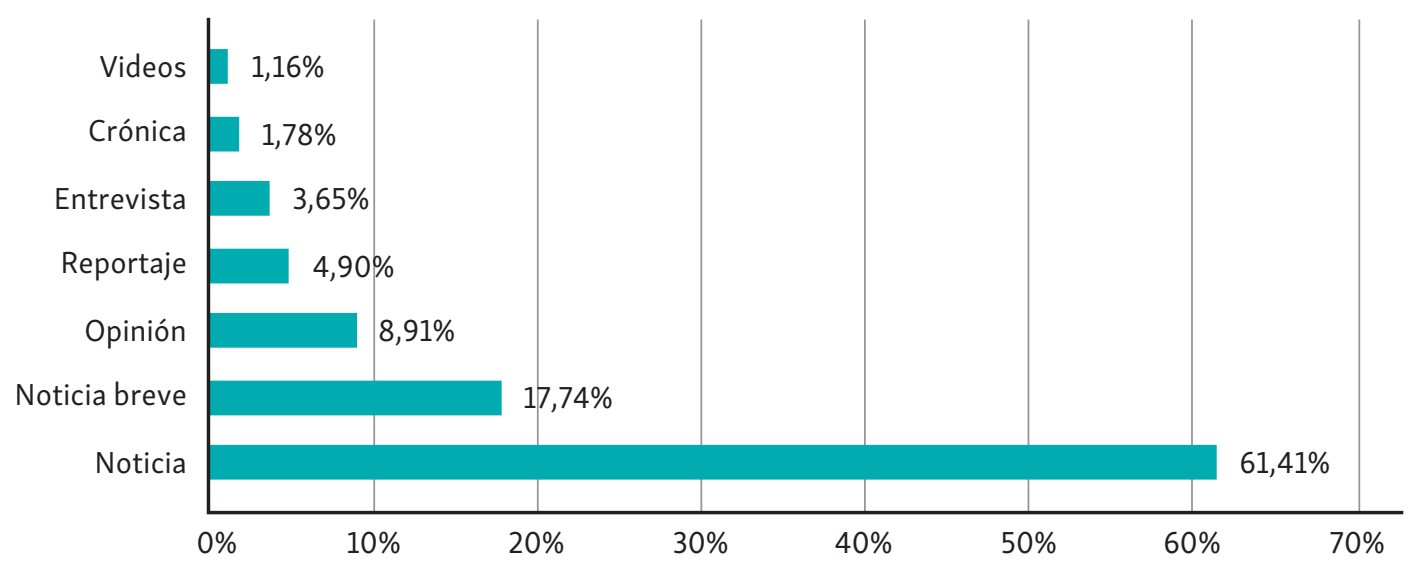

Gráfico 1. Porcentaje géneros periodísticos $(n=1122)$

Fuente: Elaboración propia.

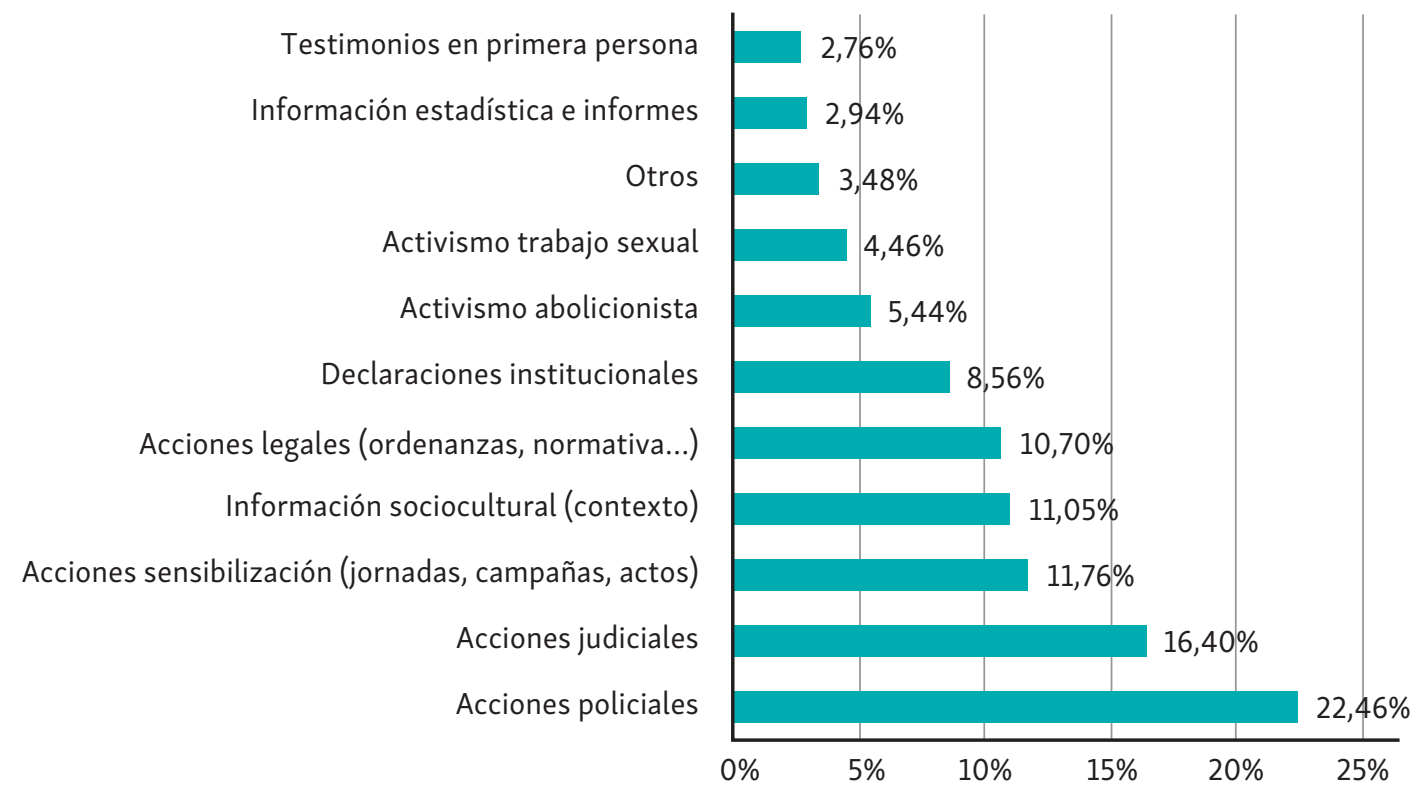

Gráfico 2. Porcentaje temáticas $(n=1122)$

Fuente: Elaboración propia.

La presencia de testimonios en primera persona, aunque minoritarios, marca un claro desplazamiento con respecto al período anterior y sigue una tendencia que arranca en 2015. Resulta significativo que $18,98 \%$ de las informaciones esté vinculado con el sindicato OTRAS. La tercera noticia más comentada (757 publicaciones) ${ }^{7}$ aborda en su titular el relevo de la directora de Trabajo "por aprobar un sindicato de prostitutas" (Cué, 2018). La quinta noticia en relevancia por el número de comentarios ${ }^{8}$ recoge las declaraciones de las portavoces de OTRAS 
en su titular: "El sindicato de prostitutas: 'Estamos constituidas y trabajando"” (Álvarez, 2018). En la muestra de 2013-2014 fue el debate sobre la regulación de la prostitución el que produjo mayor impacto y controversia.

\section{Los relatos y los actores}

Una de las tendencias observadas es el relato principal de la persecución del delito de trata que desde hace años está construyendo el imaginario mediático sobre la prostitución. Es un relato tópico que se articula en forma de noticia o de narración breve que pormenoriza una acción policial a partir de recursos que proporcionan sobre todo las Fuerzas y Cuerpos de Seguridad del Estado'. De hecho, sus gabinetes de comunicación son la fuente principal en la muestra de 2018 (20,59\%). Ese relato hegemónico se construye, en ambos casos, en torno a las mujeres denominadas prostitutas, muchas menores de edad, que son liberadas de la explotación sexual por las fuerzas del orden. En la nube de palabras que surge predomina el término prostitución, y, en torno a él, mujeres, sexual, prostitutas, trata y sindicato. Esto nos da la pauta de los relatos y actores que resultan fundamentales en esta ocasión como una continuidad y como una disrupción con respecto a la muestra anterior ${ }^{10}$.

En 2013-2014, al relato tópico de la liberación policial se suman las redes, como actores principales y como referentes sin definir frente a las mujeres o la policía. En 2018 , el proxenetismo ocupa el centro en un $38,95 \%$. Los proxenetas se presentan, como en la muestra precedente, como un actor malvado, inmigrante, perseguido por la policía y en muchos casos enjuiciado, o bien como un empresario local, dueño de locales de alterne, más honrado. Esto supone un desplazamiento que responde a las recomendaciones de las guías sobre dejar de focalizar en las mujeres.

Los clientes son actores mucho menos visibles en ambas muestras, aunque la coincidencia con una campaña del Ayuntamiento de Madrid les dio mayor visibilidad en el estudio de 2014. La demanda supuso en 2018 el eje de un 18,36\% de las piezas. Algunas abordan quiénes son los clientes, sus motivaciones o les dan voz como fuente en el debate ${ }^{11}$. Los clientes son también, en ambos estudios, uno de los focos de las medidas sancionadoras. La condición o no de inmigrante o la clase socioeconómica marcan la construcción de un imaginario amable o deplorable de estos actores.

9. Ver los estudios que identifican esta estrategia retórica o tipo de enmarcado en el ámbito internacional (Gregoriou, 2018).

10. Otra de las conclusiones de los estudios internacionales es la feminización de las informaciones y la representación estereotipada de los varones, como traficantes y clientes (Gregoriou, 2018).

11. Como el reportaje de El Mundo, que presenta diferentes perfiles bajo el paraguas de este titular: "Pago por sexo, pero no soy una bestia" (Domínguez, 2018). 


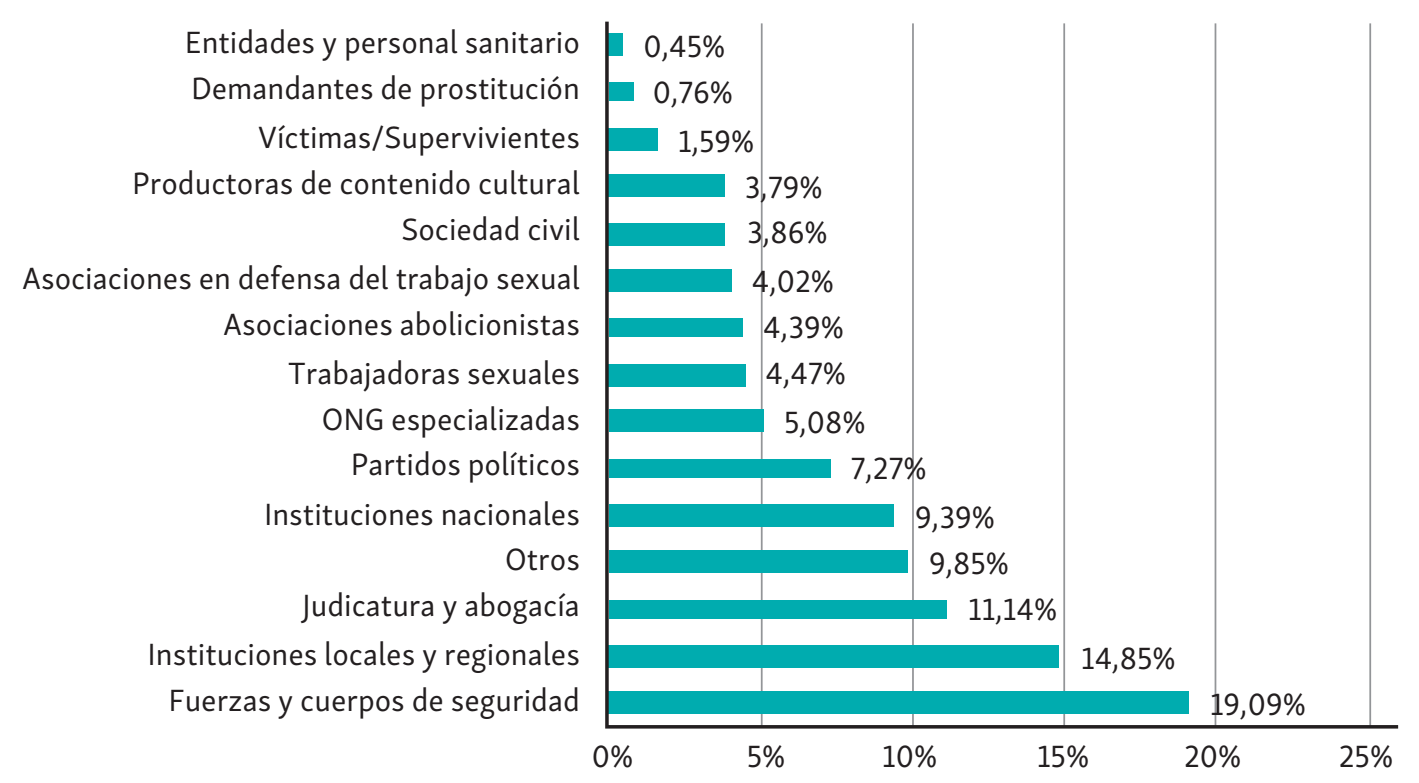

Gráfico 3. Porcentaje fuentes ( $n=1122)$

Fuente: Elaboración propia.

En $2018,12,12 \%$ piezas no citan ninguna fuente, $68.36 \%$ recurre a una sola y solo 2,5\% incorpora cuatro. Del conjunto de fuentes recogidas (1320), resultaron relevantes las instituciones locales y regionales (14,85\%), la judicatura y la abogacía $(11,14 \%)$, las instituciones nacionales $(9,39 \%)$ y sobre todo la policía y la guardia civil (19,09\%). Este hecho contribuye a mantener el relato de orden público identificado en el estudio anterior, además del relato de la legislación: medidas legislativas, ordenanzas, juicios, sentencias.

El hecho de que 213 de las 1122 piezas de la muestra de 2018 correspondan al sindicato OTRAS indica un cambio de tendencia significativo. Las figuras femeninas, marcadas, sobre todo en los relatos nacionales e internacionales de la trata, por el relato inexorable de la pobreza y la vulneración de los derechos humanos de mujeres y niñas, también adquieren el carácter de trabajadoras sexuales que reivindican sus derechos. Podemos identificar como figuras femeninas, por un lado, a víctimas de trata, esclavizadas por redes extranjeras clandestinas y rescatadas por los agentes nacionales del orden en narraciones carentes de contexto; por otro lado, a las prostitutas inmigrantes, que ejercen de modo transgresor en contextos de pobreza, ofreciendo sus cuerpos en la calle o en locales de alterne. En este escenario irrumpen las representantes del sindicato OTRAS, que se presentan como figuras empoderadas y como trabajadoras sexuales indignadas cuando no se reconocen sus derechos. Ellas propician el debate sobre el trabajo sexual y alteran las agendas monótonas y sin especialización de los medios con respecto a estos asuntos. Frente a ellas, se revitalizan las voces de las mujeres abolicionistas 
para derogar el sindicato y volver a los cauces del debate: mujeres asociadas al gobierno socialista, que aprueba y luego prohíbe el sindicato; columnistas, actrices, exprostitutas. Las fuentes se inclinan hacia el equilibrio: 53 piezas mencionan asociaciones defensoras del trabajo sexual y 58 entidades abolicionistas.

\section{Patrones de representación visual}

La mayoría de las informaciones del estudio de 2018, 73,62\%, incorporan al menos una fotografía. Solo un $8,13 \%$ de las piezas se acompaña de video.

Los patrones de representación son similares a los de 2013-2014. Priman las imágenes de fuerzas y cuerpos de seguridad del Estado, agentes, vehículos o recursos difundidos por los gabinetes de comunicación. Estas representaciones retroalimentan los relatos hegemónicos y refuerzan los imaginarios visuales de la prostitución como un universo marcado por la exclusión y la conflictividad social. Su complemento son las fotografías de mujeres de espaldas o pixeladas que activan los saberes y creencias de los públicos sobre la delincuencia y la clandestinidad. Los cuerpos femeninos se encuentran en los locales intervenidos, en pisos desmantelados, y se objetivan y cosifican al estar semiocultos, semidesnudos. Sobre todo, son mujeres solas o en grupo, generalmente racializadas, a las que se imagina migrantes y pobres; esas otras mujeres sobreexpuestas, cuyos cuerpos se fragmentan en fotografías que suelen ser robadas y donde no siempre se respeta su privacidad y anonimato.

Apenas se ilustran las noticias con fotos de demandantes de prostitución. Los clientes tampoco eran objeto de atención en la muestra anterior, si bien en esta hay algo más de presencia para poner rostro a los actores masculinos de los mercados del sexo. Otro agente masculino es la figura del proxeneta, también residual, ya que las acciones judiciales y legales en las que participan son representadas más a menudo mediante imágenes de edificios y calles.

Encontramos, como una tendencia más relevante en 2018, imágenes de actos públicos, ruedas de prensa, jornadas, etc. Entre ellas destaca como novedad la presencia de las trabajadoras sexuales como portavoces de colectivos reivindicativos. Si se presentaban de modo minoritario en el estudio de 2013-2014, asociadas a una profesión no criminalizada o glamorosa, en 2018 aparecen en eventos relacionados con el sindicato OTRAS. Esta podría considerarse la principal transformación discursiva entre ambos períodos, mientras que las piezas que se relacionan con la policía o con la prostitución mantienen las mismas tendencias. El recurso a las ilustraciones y los gráficos asociados a la información estadística continúa siendo totalmente minoritario. 


\section{DISCUSIÓN Y CONCLUSIONES}

Aunque las dos muestras son diversas en cuanto al número de medios y noticias, y por lo tanto difícilmente contrastables en algunos aspectos, su comparativa general permite extraer las tendencias de producción informativa en los medios españoles sobre prostitución y trata.

Buena parte de la información resulta homogénea en ambos períodos, ya que los medios no suelen elaborar contenidos propios, sino reproducir o ampliar los materiales de agencia, que a su vez beben de los comunicados de las fuerzas y cuerpos de seguridad del Estado, como corroboran estudios internacionales (Krsmanovic, 2018; Gregoriou, 2018). La precariedad laboral, la falta de especialización y la tematización clásica centrada en asuntos de política o economía conllevan un escaso desarrollo del periodismo de investigación, que implicaría aproximaciones más profundas (Fernández-Romero \& Simón-Carrasco, 2019).

Los medios locales no han incorporado como propio el relato de la prostitución, aunque empiezan a publicar informaciones de elaboración propia. Esta tendencia es mayor en los medios nacionales, que agendan piezas sobre trata, como El País, o prostitución, como El Confidencial, con cierto nivel de profundidad. A raíz de la polémica del sindicato OTRAS, se publicaron piezas antes excepcionales, como la cobertura de ruedas de prensa o entrevistas a trabajadoras sexuales.

En la muestra de 2018, la información sobre la trata y la explotación sexual es cada vez más frecuente. En el debate sobre prostitución se mezclan, en ocasiones, el activismo y la posición gubernamental, discusión que favorece una mayor distinción entre prostitución y trata que hace cinco años. Como apunta Clua (2015), la aparición del nuevo actor, las trabajadoras sexuales reclamando sus derechos, no hubiera podido existir sin el proceso de mediatización.

Respecto de los patrones de representación, los ejes temáticos, las voces y las líneas discursivas, hay continuidades y desplazamientos. La asimilación de los asuntos de la trata y de la prostitución, y el sesgo abolicionista, detectados en la muestra contrastiva persisten. Si bien se nota la emergencia del nuevo relato sobre la trata basado en el eje supervivientes-delito, subsiste tanto en lo verbal como en lo visual una tópica basada en el eje víctimas-redada, tradicionalmente vinculado al tratamiento de la prostitución (como sinónimo de trata).

En cuanto a las transformaciones, se aborda más el tema de los clientes y de la demanda, algo que había aparecido en las campañas de sensibilización, al mencionarse en los planes estatales y municipales la demanda de servicios sexuales como parte del problema. Respecto de las recomendaciones informativas establecidas en el II Plan Integral en línea para un enfoque adecuado a la realidad de 
la trata, respetar la imagen de las mujeres y la realidad de las víctimas, se observan algunos avances. Los tipos de pieza, más proclives a la opinión, a los reportajes en profundidad y entrevistas en primera persona marcan el principal cambio de tendencia: visibilizar testimonios de las mujeres. Las temáticas se mantienen, aunque entra con fuerza la polémica del sindicato OTRAS, que implica profundizar enlos argumentos del debate. Los medios cubren cada vez más el activismo, y por tanto las activistas abolicionistas y las pro derechos son un actor y una fuente estable en el relato, sobre todo en la información local y regional.

No se observa en ambos períodos un discurso crítico por parte de los medios, ni informaciones que cuestionen el ámbito de las políticas públicas sobre trata o prostitución. Tampoco se realizan análisis de datos de calidad, pues se suelen repetir fuentes y las cifras no se cuestionan.

Como señala Krsmanovic (2018), los marcos mediáticos dependen y dan forma a la voluntad política sobre cómo abordar este asunto. El predominio de los marcos sobre justicia criminal atestigua las ansiedades sociales y estatales acerca de las fronteras, la raza y la etnicidad, el género y la moralidad, y se aleja de las principales recomendaciones del II Plan Integral. Dar voz a esos temores ofrece grandes titulares, pero afecta a quienes son vulnerables, porque sirve para relegar el enfoque de derechos humanos, frente a los de seguridad y control migratorio. El estudio de la información nacional, regional y local sobre prostitución y trata en España invita a una compresión integral de ambos asuntos y a dar mayor cabida a los testimonios como garantía de activación de una transformación social a partir de la implicación de los públicos en ellos como parte de su responsabilidad.

\section{FINANCIAMIENTO}

Proyecto I+D Problemas públicos y controversias: diversidad y participación en la esfera mediática (CSO2017-82109-R) (2018-21), Ministerio de Ciencia e Innovación, gobierno de España.

Proyecto "Simposio La representación de la trata con fines de explotación sexual en el espacio público". PAC18 exp.:29/2ACT/18. Convocatoria competitiva IMIO: subvenciones para realizar postgrados de estudios de género y actividades del ámbito universitario relacionadas con la igualdad de oportunidades entre mujeres y hombres (2018). Instituto de la Mujer y para la Igualdad de Oportunidades, Ministerio de la Presidencia, Relaciones con las Cortes e Igualdad, España. 


\section{REFERENCIAS}

Alvarado-López, M. C. \& Justo-von-Lurzer, C. (2019). Prostitución y medios de comunicación: de la controversia a la innovación social (Prostitution and mass media: from the controversy to the social innovation). Revista Mediterránea de Comunicación/Mediterranean Journal of Communication, 10(1), 93-94. https://doi.org/10.14198/MEDCOM2019.10.1.26

Allwood, G. (2018). Agenda setting, agenda blocking and policy silence: Why is there no EU policy on prostitution? Women's Studies International Forum, 16. https://doi.org/10.1016/j.wsif.2018.06.004

Álvarez Pousa, L., Castro Vásquez, O., Cuevas Domínguez, I., \& Puñal Rama, A. (2010). A representación da prostitución nos medios de comunicación galegos. Proxecto de investigación. Santiago de Compostela, Spain: Colexio Profesional de Xornalistas de Galicia. Retrieved from http://xornalistas.gal/web/uploads/materiais_biblioteca_adxuntos/ adxunto/85e/54d0a74061-prostitucion-medios-informe-1.pdf

Álvarez, P. (2018, September 21). “El sindicato de prostitutas: 'Estamos constituidas y trabajando'” (“The prostitute union: 'We are constituted and working'”). El País. Retrieved from: https://elpais.com/sociedad/2018/09/21/actualidad/1537543646_262587.html

Austin, R. \& Farrell, A. (2017). Human trafficking and the media in the United States. Oxford Research Encyclopedia of Criminology and Criminal Justice. https://doi.org/10.1093/acrefore/9780190264079.013.290

Ayuntamiento de Madrid. (2014). Estudio sobre el tratamiento publicitario e informativo de la prostitución y la trata de seres humanos con fines de explotación sexual en los medios de comunicación: Diagnóstico, y propuestas de actuación (Study on the Advertising and Informational Treatment of Prostitution and Human Trafficking for the Purpose of Sexual Exploitation in the Media: Diagnosis and Proposals for Action). Retrieved from https://www.bienestaryproteccioninfantil.es/fuentesl.asp?sec=18\&subs=183\&cod=2924\&page=

Ayuntamiento de Barcelona \& Asociación de Mujeres Periodistas de Cataluña. (2016). Análisis del tratamiento en los medios de comunicación de la trata con fines de explotación sexual $y$ de la prostitución (Analysis of the Treatment in the Media of Trafficking for the Purpose of Sexual Exploitation and Prostitution). http://www.adpc.cat/new_site/wp-content/ uploads/2016/05/Prostitucion_2015_def_analisi_ES.pdf

Benítez-Eyzaguirre, L. (2017). La imagen audiovisual de la prostitución en las cadenas de televisión en España (The audiovisual image of prostitution in Spain's television networks). Estudios Sobre el Mensaje Periodístico, 23(1), 325-341. https://doi.org/10.5209/ESMP.55599

Bernstein, E. (2007). Buying and selling the 'girlfriend experience'. In M. Padilla (Ed.), Love and globalization: transformations of intimacy in the contemporary world (pp. 186-202). Vanderbilt University Press.

Bozorova, M. (2019). Coverage of the Problem of Human Trafficking in the Media: Content Analysis of Materials of Editions of Russian Federation. ESSACHESS-Journal for Communication Studies, 12(23), 53-75. Retrieved from https://www.essachess.com/index.php/jcs/article/view/444 
Brosa, J. \& Medina, P. (2012). Representación de la prostitución inmigrante en la prensa. El caso de las prostitutas del barrio del Raval de Barcelona (Representation of immigrant prostitution in the press. The case of the prostitutes in the Raval district of Barcelona). Estudios Sobre el Mensaje Periodístico, 18(1), 259-273. https://doi.org/10.5209/ revESMP.2012.v18.n1.39369

Calvo, F. (2001). Apuntes para un análisis de prensa (Notes for a press analysis). In E. Bonelli Jáudenes, M. Ulloa Jiménez, F. Calvo Ocampo, I. López Méndez, \& L. Oso Casas. Tráfico de inmigración de mujeres en España: colombianas y ecuatorianas en los servicios domésticos $y$ sexuales (Immigration trafficking of women in Spain: Colombians and Ecuadorians in domestic and sexual services) (pp. 51-63). ACSUR-Las Segovias.

Cefaï, D. (2016). Publics, problèmes publics, arènes publiques... Que nous apprend le pragmatisme? Questions de communication, 2(30), 25-64.

https://doi.org/10.4000/questionsdecommunication.10704

Clua, A. (2015). La batalla simbólica de las prostitutas. El papel de la comunicación. Revista Internacional de Comunicación y Desarrollo (RICD), 1(1), 139-150. Retrieved from http://www.usc.es/revistas/index.php/ricd/article/view/2352

Cué, C. E. (2018, September 3). Relevada la directora de Trabajo por aprobar un sindicato de prostitutas (The Director of Labor relieved for approving a union of prostitutes). El País. Retrieved From https://elpais.com/politica/2018/09/03/actualidad/1535979318_955992.html

Delegación del Gobierno Contra la Violencia de Género. (2015). II Plan Integral contra la trata con fines de explotación sexual (II Comprehensive Plan Against Trafficking for Sexual Exploitation Purposes) (2015-2018). Retrieved from https://violenciagenero.igualdad. gob.es/planActuacion/planContraExplotacionSexual/docs/Plan_Integral_Trata_18_ Septiembre2015_2018.pdf

Devillard, V. \& Le Saulnier, G. (2015). Le problème public de la prostitution aux marges des arènes publiques numériques. Luttes interprétatives et mobilisations pro-droits contre l'offensive abolitionniste sur le web (The public problem of prostitution on the fringes of digital public arenas. Interpretative struggles and pro-rights mobilizations against the abolitionist offensive on the web). Journal des antropologues, 142-143, 203-226. https://doi.org/10.4000/jda.6279

Di Ronco, A. (2017). Disorderly or simply ugly? Representations of the local regulation of street prostitution in the Italian press and their policy implications. International Journal of Law, Crime and Justice, 52, 10-22. https://doi.org/10.1016/j.ijlcj.2017.09.004

Domínguez, C. (2018, June 18). Hablan los clientes de la prostitución: ‘Pago por sexo pero no soy unabestia' ("Prostitution clients speak up:'I pay for sex butI'm notabeast").ElMundo. Retrieved from https://www.elmundo.es/papel/historias/2018/06/18/5b225bd546163f17be8b45ad.html

Easterbrook-Smith, G. (2018) "Illicit drive-through sex", “Migrant Prostitutes", and "Highly Educated Escorts": Productions of 'acceptable' sex work in New Zealand news media 2010 - 2016 (Doctoral dissertation, University of Wellington). Retrieved from http://hdl.handle.net/10063/6989 
Fernández-Romero, D. \& Simón-Carrasco, P. (2019). La información sobre prostitución y trata como escenario de disputa: percepciones de las estrategias de enunciación desde los actores implicados (Information around prostitution and trafficking as a dispute stage: perceptions of enunciation strategies from involved actors). Revista Mediterránea de Comunicación, 10(1), 161-172. https://doi.org/10.14198/MEDCOM2019.10.1.10

Fraser, N. (2012). Reflexiones en torno a Polanyi y la actual crisis capitalista (Reflections on Polanyi and the current capitalist crisis). Papeles de relaciones ecosociales y cambio global, 118, 13-28. Retrieved from https://www.fuhem.es/wp-content/uploads/2018/12/ reflexiones_sobre_Polanyi_y_la_actual_crisis_capitalista_N.Fraser.pdf

Friedman, B. \& Johnston, A. (2018). Editors' Note: Introduction to the Special Issue. Journal of Human Trafficking, 4(1), 1-5. https://doi.org/10.1080/23322705.2017.1423442

Gregoriou, C. (Ed.) (2018). Representations of Transnational Human Trafficking Present-day News Media, True Crime, and Fiction. Palgrave McMillan.

Gregoriou, C. \& Ras, I. A. (2018). Call for Purge on the People Traffickers: An Investigation into British Newspapers Representation of Transnational Human Trafficking, 2000-2016. In C. Gregoriou (Ed.), Representations of Transnational Human Trafficking Present-day News Media, True Crime, and Fiction (pp. 25-29). Palgrave McMillan.

Guerrero Bernal, J. C., Márquez Murrieta, A., Nardacchione, G., \& Pereyra, S. (2018). Problemas públicos. Controversias y aportes contemporáneos. Estudio Introductorio (Public problems. Contemporary controversies and contributions. Introductory Study). In J. C. Guerrero Bernal, A. Márquez Murrieta, G. Nardacchione, \& S. Pereyra (Coords.), Problemas públicos: controversias y aportes contemporáneos (Public problems. Contemporary controversies and contributions) (pp. 9-54). Instituto de Investigaciones Dr. José María Luis Mora.

Gutiérrez García, A. (2013). La actualidad del abordaje de la prostitución femenina en la prensa diaria española (The Current Situation in the Tackling of Female Prostitution in the Spanish Daily Press). Estudios Sobre el Mensaje periodístico, 19, 823-831. https://doi.org/10.5209/rev_ESMP.2013.v19.42165

Heim, D. (2012). Más allá del disenso: los derechos humanos de las mujeres en los contextos de prostitución (Beyond dissent: the human rights of women in prostitution contexts). Derechos y Libertades: revista de filosofía del derecho y derechos humanos, 26(1), 297-327. https://doi.org/10.1400/216303

Hepp, Andreas (2020). Deep Mediatization. Routledge.

Hunt, S. \& Hubbard, B. (2015). The representation of sex workers in South African media: Danger, morals and human rights. Stellenbosch Papers in Linguistics Plus, 46, 25-49. https://doi.org/10.5842/46-0-618

Justo von Lurzer, C. (2014). Sexualidades en foco. Representaciones televisivas de la prostitución en la Argentina (Sexualities in focus. Television representations of prostitution in Argentina). Sexualidades. Center for Lesbian and Gay Studies Graduate Center, 11, 1-57. Retrieved from https://ri.conicet.gov.ar/handle/11336/35849

Kempadoo, K. (2003). Globalizing Sex Workers' Rights. Canadian Woman Studies, 22(3-4), 143-150. 
Kempadoo, K., Sanghera, J., \& Pattanaik, B. (2005). Trafficking and prostitution reconsidered: new perspectives on migration, sex work, and human rights. Paradigm Publishers.

Kohlbacher, F. (2006). The Use of Qualitative Content Analysis in Case Study Research. Forum Qualitative Sozialforschung, 7(1). https://doi.org/10.17169/fqs-7.1.75

Krsmanovic, E. (2018). Different Alfabet Some Story? Media Framing of Human Trafficking for Sexual Exploitation in British, Dutch and Serbian Media (Doctoral dissertation, Utrecht University). Retrieved from https://dspace.library.uu.nl/handle/1874/371384

Marres, N. (2015). Why Map Issues? On Controversy Analysis as a Digital Method. Science Technology and Human Values, 40(5), 655-686. https://doi.org/10.1177/0162243915574602

Marres, N. (2017). Digital Sociology. The reinvention of social research. Polity Press.

Núñez Puente, S. \& Fernández Romero, D. (2015). Identity construction of the subject victim of domestic violence: fetishism, aesthetization and public identity. Teknokultura, 12(2), 267-284. https://doi.org/10.5209/rev_TK.2015.v12.n2.49506

Papadouka, M., Evangelopoulos, N., \& Ignatow, G. (2016). Agenda setting and active audiences in online coverage of human trafficking. Information, Communication \& Society, 19(5), 655-672. https://doi.org/10.1080/1369118X.2016.1139615

Piña, R. (2015, April 14). Ciudadanos apuesta por legalizar la prostitución y la incorporación al mundo laboral como autónomos (Ciudadanos is committed to legalizing prostitution and joining the world of work as freelancers). El Mundo. Retrieved from https://www.elmundo.es/espana/2015/04/14/552c394fca4741435c8b456c.html

Puñal, A. B. (2015). Presenza e ausencia das mulleres na prensa. Análise do tratamento da prostitución en El País e ABC (Women's presence and absence in the press. Analysis of prostitution treatment in El País and $A B C$ ) (Doctoral dissertation, Universidade de Santiago de Compostela). Retrieved from http://dspace.usc.es/handle/10347/13376.

Puñal Rama, B. \& Tamarit, A. (2017). La construcción mediática del estigma de prostituta en España (The Mediatized Construction of the Stigma against the Prostitute in Spain). Ex æquo, (35), 101-123. https://doi.org/10.22355/exaequo.2017.35.07

Raphael, J. (2017). Returning Trafficking Prevalence to the Public Policy Debate: Introduction to the Special Issue. Journal of Human Trafficking, 3(1), 1-20.

https://doi.org/10.1080/23322705.2017.1280314

Rusnac, L. (2019). Human trafficking as a social phenomenon and media event: contextualization and speech. International Journal of Communication Research, 9(2), 136-140.

Sabsay, L. (2016). The political imaginary of sexual freedom. Subjectivity and Power in the New Sexual Democratic Turn. Palgrave Macmillan. https://doi.org/10.1057/978-1-137-26387-2

Sagredos, C. (2019). The representation of sex work in the Greek Press. A corpus-assisted critical discourse analysis. Journal of Language and Sexuality, 8(2), 166- 194. https://doi.org/10.1075/jls.18012.sag

Saiz-Echezarreta, V. (2019). Mediatización de las controversias públicas: a propósito de la campaña sobre prostitución Hola Putero (Mediatization of public controversies: analysis of the HolaPutero campaign against prostitution). Revista Mediterránea de Comunicación/Mediterranean Journal of Communication, 10(1), 95-115. https://www.doi.org/10.14198/MEDCOM2019.10.1.19 
Saiz Echezarreta, V. \& Martínez Pérez, E. (2018). Incidencia del movimiento pro derechos del trabajo sexual en el discurso mediático (Incidence of movement for sex work in media discourse). In A. M. Olmo Gascón. Diversidad Sexual y libertad reproductiva de las mujeres en la cultura de la producción y del consumo (Sexual diversity and reproductive freedom of women in the culture of production and consumption) (pp.39-70). Editorial Comares.

Saiz-Echezarreta, V., Peralta García, L., \& Mártinez Pérez, E. (2018). Alianzas de actores y construcción de una arena pública a través de la estrategia transmedia. Análisis del proyecto Chicas Nuevas 24 Horas (Alliances of actors and construction of a public arena through transmedia strategy. Analysis of the New Girls 24 Hours Project). Teknokultura. Revista de Cultura Digital y Movimientos Sociales, 15(2), 207-222. https://doi.org/10.5209/TEKN.59548

Saiz-Echezarreta, V., Alvarado, M., \& Gómez-Lorenzini, P. (2018). Incidencia política de las campañas contra la trata: Un relato controvertido (Advocacy of trafficking campaigns: A controversy story). Revista Comunicar, 26(55), 29-38. https://doi.org/10.3916/C55-201803

Sanford, R., Martinez, D. E., \& Weitzer, R. (2016). Framing Human Trafficking: A Content Analysis of Recent U.S. Newspaper Articles. Journal of Human Trafficking, 2(2), 139-155. https://doi.org/10.1080/23322705.2015.1107341

Snow, J. (2017). A Content Analysis on the Internet News Media and the Growth of Human Trafficking. The School of Communication and the Graduate School of Point Park University.

Sobel, M. (2016). Confronting sex trafficking: Gender depictions in newspaper coverage from the former Soviet republics and the Baltic states. European Journal of Communication, 31(2), 152-168. https://doi.org/10.1177/0267323115612211

Souza, B. \& Carvalho, C. (2012). Jornalismo e homofobia no Brasil: mapeamento e reflexões (Journalism and homophobia in Brazil: mapping and reflections). Intermeios.

Strega, S., Janzen, C., Morgan, J., Brown, L., Thomas, R., \& Carriere, J. (2014). Never innocent victims: street sex workers in Canadian print media. Violence Against Women, 20(1), 6-25. https://doi.org/10.1177/1077801213520576

Venturini, T. (2012). Building on faults: How to represent controversies with digital methods. Public Understanding of Science, 21(7), 796-812. https://doi.org/10.1177/0963662510387558

Vicente Mariño, M. (2009). Desde el análisis de contenido hacia el análisis del discurso. La necesidad de una apuesta decidida por la triangulación metodológica (From content analysis to the speech analysis. The need for a solution decided by methodological triangulation. In F. Sierra (Coord.) Iberoamérica: comunicación, cultura y desarrollo en la era digital (Iberoamerica: communication, culture and development in the digital age). Universidad de Sevilla. https://bit.ly/32nJHn5

Zelizer, V. A. (2011). Economic Lives: How Culture Shapes the Economy. Princeton University Press. 


\section{SOBRE LAS AUTORAS}

vanesa SAIZ echezarReta, Profesora Contratada Doctora, Facultad de Comunicación, Universidad de Castilla-La Mancha, España. Docente en Teoría del Periodismo, Métodos de investigación y Cultural digital. Investigadora del Grupo de investigación Semiótica, comunicación y cultura (UCM), especializada en análisis sociocultural con perspectiva semiótica, dimensión afectiva de movimientos sociales y estudios de género. Producción científica sobre mediatización de problemas públicos, controversias y participación, centrada en el estudio de caso sobre la prostitución.

iD https://orcid.org/0000-0003-1700-0296

DiANA FERNÁNDEZ RomERo, Profesora Contratada Doctora, Facultad de Ciencias de la Comunicación, Universidad Rey Juan Carlos, Madrid, España. Docente en Teorías de la Información y directora del Título Especialista en Prevención y Sensibilización de las Violencias Sexuales y de Género desde un enfoque multidisciplinar (URJC). Miembro del grupo de investigación de alto rendimiento sobre comunicación, sociedad y cultura GICOMSOC. Producción científica sobre comunicación, género, testimonio ético y mediatización de la rabia femenina.

iD https://orcid.org/0000-0003-2692-4474

MARía CRUz ALVARAdo lópez, Profesora Titular, Facultad de Ciencias Sociales, Jurídicas y de la Comunicación, Universidad de Valladolid, España. Docente en Teoría e Historia de la Publicidad y coordinadora del Máster en Comunicación con Fines Sociales, Estrategias y Campañas; de la Universidad de Valladolid. Miembro del Grupo de Investigación en Comunicación Audiovisual e Hipermedia (GICAVH), actualmente trabajando en el proyecto INTERNÉTICA. Producción científica sobre publicidad social, historia de la publicidad en España y comunicación con fines sociales con perspectiva de género.

(iD) https://orcid.org/0000-0002-1659-7249 